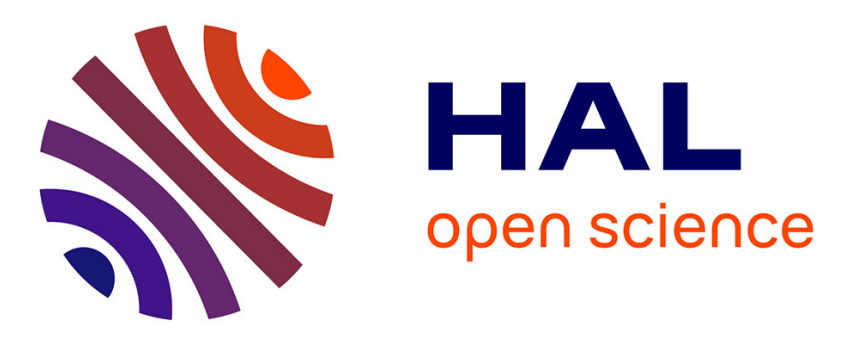

\title{
An electret film-based triboelectric nanogenerator with largely improved performance via a tape-peeling charging method
}

Hanlu Zhang, Shan Feng, Delong He, Yiguo Xu, Minhao Yang, Jinbo Bai

\section{- To cite this version:}

Hanlu Zhang, Shan Feng, Delong He, Yiguo Xu, Minhao Yang, et al.. An electret film-based triboelectric nanogenerator with largely improved performance via a tape-peeling charging method. Nano Energy, 2018, 48, pp.256 - 265. 10.1016/j.nanoen.2018.03.051 . hal-01841854

\section{HAL Id: hal-01841854 \\ https://hal.science/hal-01841854}

Submitted on 21 May 2020

HAL is a multi-disciplinary open access archive for the deposit and dissemination of scientific research documents, whether they are published or not. The documents may come from teaching and research institutions in France or abroad, or from public or private research centers.
L'archive ouverte pluridisciplinaire HAL, est destinée au dépôt et à la diffusion de documents scientifiques de niveau recherche, publiés ou non, émanant des établissements d'enseignement et de recherche français ou étrangers, des laboratoires publics ou privés. 


\title{
An electret film-based triboelectric nanogenerator with largely improved performance via a tape-peeling charging method
}

Hanlu Zhang, Shan Feng, Delong He*, Yiguo Xu, Minhao Yang, Jinbo Bai*

Laboratoire Mécanique des Sols, Structures et Matériaux (MSSMat), CNRS UMR 8579,

Ecole CentraleSupélec, Université Paris-Saclay, 8-10 rue Joliot-Curie, 91190 Gif-sur-Yvette, France

${ }^{*}$ Corresponding authors: jinbo.bai@centralesupelec.fr; delong.he@centralesupelec.fr

\begin{abstract}
Harvesting energy from the environment is a sustainable solution for powering decentralized sensor networks, Internet of Things systems, etc. In this work, a triboelectric nanogenerator (TENG) based on the fluorinated ethylene propylene (FEP) electret film is investigated to generate electricity from mechanical motions in the environment, and its working principle is explained with a variable capacitance model. For the first time, the validity of this model is verified with a capacitor discharge curve fitting method. Based on this model, the maximum output energy of the TENG per working cycle is calculated, which could be useful for comparing the property of TENGs working under different environmental conditions. An adhesive tape-peeling process is innovatively used to charge the FEP electret film, which is facile and effective for increasing the charge amount on the film without using any complex equipment. Through this method, the surface potential of a $4 \mathrm{~cm} \times 4 \mathrm{~cm}$ FEP film is improved from $-360 \mathrm{~V}$ to
\end{abstract}


$-2850 \mathrm{~V}$, the output negative voltage peak of the same-sized TENG is improved from -83 $\mathrm{V}$ to $-363 \mathrm{~V}$, and for a wind-driven $8 \mathrm{~cm} \times 8 \mathrm{~cm}$ sized TENG, its average output power to charge a $10 \mu \mathrm{F}$ capacitor is improved from $2.5 \mu \mathrm{W}$ to $19.8 \mu \mathrm{W}$. The ability of this TENG in generating electricity from low-frequency human motions to directly light up light emitting diodes (LEDs) is also demonstrated.

Keywords: energy harvesting; triboelectric nanogenerator; FEP electret film; variable capacitance model; capacitor discharge curve fitting; tape-peeling charging method;

\section{Introduction}

Miniature energy harvesters are good choices to replace batteries in decentralized sensor networks, wearable or implantable devices, "Internet of Things" systems with lots of nodes, etc., where power consumption is low in each node but regular maintenance becomes difficult [1-3]. In the ambient environment, ubiquitous irregular mechanical motions such as wind, ocean waves, human motions, and solid vibrations contain sustainable huge kinetic energy $[4,5]$. The problem is how to convert these diverse forms of mechanical energy into electricity $[6,7]$. Conventional miniature mechanical energy harvesters are based on electromagnetic induction [8,9], piezoelectric effect [10-13], or electrostatics [14-18]. Since the year of 2012 [19], a new type of energy harvester named triboelectric nanogenerator (TENG) is intensively exploited as an effective way to harvest kinetic energy and generate electric energy from almost all kinds of mechanical motions [20-30].

The definition of TENG was given as "a device that converts mechanical energy into electricity using the coupling effects between triboelectrification and electrostatic 
induction through the contact-separation or relative sliding between two materials that have opposite tribo-polarity" [31]. A typical TENG should contain at least one dielectric layer retaining electrical charges for electrostatic induction. Such a dielectric with retained charges is termed as an electret [32]. It has been noted that the surface charge density of the electret layer significantly influences the output of the TENG [5, 33-37]. Nevertheless, the working principle of TENG still deserves to be further discussed and verified.

On the other hand, the electromechanical response and charge dynamics of electret films have been continuously studied [38-46]. Two most widely used methods to make electrets are thermal and corona charging methods [47-52]. For both methods, high voltage sources and assorted apparatuses are indispensable. While for TENGs, triboelectrification, which is the charge transfer phenomenon between two surfaces or fluid/solid interfaces after contact and separation $[26,53,54]$, is used to charge dielectrics without any equipment [32]. Nevertheless, the triboelectric charge density cannot be directly controlled or varied without changing the materials employed [34]. Therefore, some researchers combine the TENG with the corona charging electrets [34, 55, 56], however, this makes the high voltage source indispensable again, which complicates the performance improvement of TENGs.

Here, we report a TENG based on fluorinated ethylene propylene (FEP) electret film which is charged through a controllable tape-peeling tribo-charging method instead of using any complex equipment. Firstly, we illuminate its working principle with a variable capacitance model. And we experimentally verify this model for the first time with the 
capacitor discharge curve fitting method. Secondly, to charge the electret FEP film, we innovatively use the adhesive tape-peeling process, which is facile and effective. The surface potential of a $4 \times 4 \mathrm{~cm}^{2}$ sized FEP film was improved from $-360 \mathrm{~V}$ to $-2850 \mathrm{~V}$, the output voltage and power of the TENG was thereby obviously enhanced. In addition, we also demonstrate harvesting energy from wind and human motions with this TENG. This work provides better comprehensions to the working principle of TENGs, instructions for improving the performance of TENGs, and a low-cost, facile, and effective method to charge electrets for high-performance TENGs and other applications.

\section{Experimental}

\subsection{Materials and fabrication processes}

To fabricate the TENG, a piece of $100 \mu \mathrm{m}$-thick FEP film was coated with silver paste on one side as an electrode, while the other side naked. A piece of Al foil was utilized as the other electrode. These two parts were aligned and attached to two objects with relative reciprocating motions that can drive them to contact and separate repeatedly, with the naked side of the FEP film faced with the Al foil. The back electrode on the FEP film and the Al foil electrode were connected through external electric loads, and a part of the kinetic energy in the relative motion of these two parts was converted into electric energy and transmitted to electric loads. For the tape-peeling process, a roll of commercial adhesive tape (Lyreco, polypropylene substrate with acrylic adhesive) was used to be pasted on and peeled off the FEP film. For the wind-driven energy harvesting, a piece of Al foil was covered on a horizontal acrylic plate, and a piece of FEP film a little smaller than the Al foil was fixed above the Al foil with back electrode upward. A 
slit of $3 \mathrm{~mm}$ height was left between one corner of the FEP film and the Al foil, through which wind was blown in to make the FEP film vibrate.

\subsection{Measurements}

To drive the TENG, a linear motor system (shown in Fig. S1, Supporting Information) was employed to provide periodic forward and backward motions. A $4 \mathrm{~cm}$ $\times 4 \mathrm{~cm}$ sized FEP film was attached to the moving block of the linear motor, with the naked side outward, and a same sized Al foil was attached to a fixed plate faced with the FEP film. During all tests, the moving speed of the linear motor in both directions was set as $0.2 \mathrm{~m} / \mathrm{s}$, and both the acceleration and deceleration set as $10 \mathrm{~m} / \mathrm{s}^{2}$. The electrode at the backside of the FEP film and the Al foil was connected to the positive and negative node of a $100 \mathrm{M} \Omega$ high impedance probe respectively, transmitting output voltage to an oscilloscope (Wavejet 354A, Lecroy). The output current and transferred charge amount were recorded with a Keithley 6514 electrometer. The surface potential of FEP film was monitored with a surface potential meter (USSVM2, AlphaLab, Inc.), with the probe located $5 \mathrm{~mm}$ above the measured surface.

\section{Result and discussion}

\subsection{Working principle of the TENG}

Fig. 1(a) depicts the cross-section structure and one working cycle of the TENG. To better reveal the core working principle, a mechanical push-button switch was added in the external circuit to assimilate its working cycle with the "prime, wait, flight, and conversion" cycle in some electrostatic micropower generators [57]. The switch was manually controlled. In the four stages shown in Fig. 1(a), the switch was only closed at 
stage (i) when the counter electrode stayed at the distance $x_{1}$ from the FEP film, and at stage (iii) when the counter electrode contacted with the FEP film. During the transition period between stage (i) and (iii), the counter electrode and the FEP film relatively moved, the switch was kept off. The switch-on state in stage (i) and (iii) started soon after the relative motion stopped, ended after the voltage peaks were fully measured, and then the relative motion driven by the linear motor began again. Under this cycle, electrostatic energy harvesters can get the maximum efficiency [58]. It should be noted that unlike most of micropower electrostatic generators [14, 57, 59], no external voltage or charge supply is needed during the working cycle of the TENG, this is the advantage of TENGs and other electret-based generators over conventional electrostatic energy harvesters. Detailed explanations about the working principle and the working cycle of this TENG are given in the following of the section.

Firstly, since FEP is a strongly triboelectric negative and insulating material [58], the FEP film got some negative charges during production and processing [60]. Under the electrostatic induction force derived from these negative charges, electrons in the back electrode (silver paste at the backside of the FEP film) will flow into other conductive objects touching with it, making the back electrode positively charged. The negative charges reserved in the FEP film can be equivalent as it has an effective surface charge density $-\sigma$ on its naked surface. At charge equilibrium state, the induced charge density on the back electrode will be $\sigma$ [32]. When a piece of Al foil (Counter electrode) was placed in face with the naked side of the FEP film, two planar capacitors in parallel connection were formed (schemed in Fig. 1(b)). One variable capacitor $C_{x}$ was formed by 
the air gap between the counter electrode and the FEP film $\left(C_{x}\right.$ variable with the gap distance $x$ ), while the other one $C_{f}$ with fixed value formed by the FEP film (thickness $d_{f}$ ), if regarding the naked FEP surface as the common electrode of $C_{x}$ and $C_{f}$. Then, when the counter electrode was connected to the back electrode through a closed-loop external circuit, electrons flowed from it to the back electrode, compensating part of positive charges there, making it also get positive charges. This parallel-connected capacitance configuration is used in our model, instead of series-wound capacitance models established in other reports [61]. The reason is explained in Supplementary Note 1 and Fig. S2 in the Supporting Information.

Under this external closed-circuit condition at a certain $x$ value of $x_{1}\left(x_{1}>0\right)$ for a spell, electrostatic equilibrium was established between the counter and back electrodes (stage (i) in Fig. 1(a)). If both $x_{1}$ and $d_{f}$ are much smaller than the side length of the FEP film, infinite plate capacitor assumption can be applied [60]. Taking the naked FEP surface as the zero-potential reference plane, with the charge conservation law and the electrostatic equilibrium condition, there are:

$$
\begin{aligned}
& Q_{x l}+Q_{b}=Q_{f} \\
& Q_{x l} / C_{x l}=Q_{b} / C_{f}
\end{aligned}
$$

where $Q_{x l}, Q_{b}$, and - $Q_{f}$ is the charge amount on the counter electrode, the back electrode, and the FEP film respectively. Merging these two equations, we get:

$$
Q_{b}=C_{f} Q_{f} /\left(C_{x l}+C_{f}\right)
$$

Then the switch was disconnected, and the counter electrode approached the FEP film under this open-circuit condition (stage (ii)), $x$ decreased from $x_{1}$ to $x_{2}\left(0<x_{2}<x_{1}\right)$, no 
charge transferred between the two electrodes during this process, $Q_{x l}$ and $Q_{b}$ kept unchanged, and $C_{f}$ also kept constant, but $C_{x}$ would increase to $C_{x 2}$ according to $C_{x}=$ $S \varepsilon_{0} / x$, where $S$ is the area of the FEP film, $\varepsilon_{0}$ is the absolute dielectric constant. Thus, the potential equilibrium between the two electrodes was broken, the back electrode held a potential difference with the counter electrode as:

$$
V_{o c}=Q_{b} / C_{f}-Q_{x 1} / C_{x 2}
$$

Further, if the counter electrode touched the FEP film surface (stage (iii)), $C_{x}$ disappeared, the open-circuit potential difference became:

$$
V_{o c-f}=Q_{b} / C_{f}=Q_{f} /\left(C_{x l}+C_{f}\right)
$$

The switch was then closed, this potential difference became an output voltage, electrons flowed from the counter electrode to the back electrode until $Q_{b}$ is all transferred to the counter electrode. The charge amount on the counter electrode and FEP film became $Q_{f}$ and $-Q_{f}$ respectively, new electrostatic equilibrium was obtained. This process can be regarded as the discharge of $C_{f}$ with charge amount of $Q_{b}$. The released electric energy during this process is:

$$
W_{f}=0.5 Q_{b}^{2} / C_{f}=0.5 C_{f} V_{o c-f}{ }^{2}=0.5 C_{f} Q_{f}^{2} /\left(C_{x l}+C_{f}\right)^{2}
$$

Afterwards, the switch was disconnected, the counter electrode and the FEP film were separated under the open-circuit condition, an electric potential difference was produced between these two surfaces with opposite charges. When the counter electrode was moved back to the same position as $x_{1}$, the switch was then closed, part of positive charges would flow back from the counter electrode to the back electrode, outputting an opposite voltage in comparison with stage (iii), until the electrostatic equilibrium was 
established again as the original stage (i). This process can be regarded as that $C_{x}$ with charge amount of $Q_{f}$ discharged a charge amount of $Q_{b}$ to $C_{f}$ to get potential balance. Taking consideration of the charge transfer direction, the open circuit voltage peak of this process would be:

$$
V_{o c-x l}=-Q_{f} / C_{x l}
$$

The released electric energy of this process is:

$$
W_{x l}=0.5\left[Q_{f}^{2} / C_{x l}-Q_{f^{2}} /\left(C_{x l}+C_{f}\right)\right]=\left(1+C_{f} / C_{x l}\right) W_{f}
$$

A whole working cycle was then finished. In such a working cycle, the TENG releases the electric energy of $W_{f}+W_{x I}$ to the external circuit. Multiple working cycles can take place if a periodical mechanical motion drives the counter electrode and the FEP film contact and separate repeatedly.

This working process can be concluded as Fig. 1(c). Periodic mechanical motions make the air gap between the counter electrode and the FEP film vary, changing the capacitance formed by this air gap and breaking the electrostatic equilibrium state in the TENG. Charges flow between the counter electrode and the back electrode through the external circuit to rebuild the equilibrium state, outputting electricity to the external load. The output voltage can be monitored with the oscilloscope with high input resistance. Owing to the high input resistance, charges need some time to be fully transferred, therefore the voltage can be measured even without the switch in the circuit. A typical voltage signal measured with the oscilloscope from the TENG during a working cycle without a switch is also presented in Fig. 1(c). Some other electret-based energy harvesters used the similar working process $[48,62,63]$. 


\subsection{Verification of the variable capacitance model}

To verify the variable capacitance model presented above, $C_{f}$ and $C_{x l}$ need to be known. Here we use the capacitor discharge curve fitting method to calculate $C_{f}$ and $C_{x l}$ from measured voltage peaks. Briefly, for a capacitor with a capacitance of $C$ and an initial voltage $\mathrm{V}_{0}$, during its discharge process with a resistance $R$ in series connection, the voltage changes with time as follows:

$$
V(t)=\mathrm{V}_{0} \exp (-t / R C)
$$

Where $t$ represents the time passed since the discharge begins, and all parameters are in SI units. This method was demonstrated by fitting discharge curves of a commercial 0.47 $\mu \mathrm{F}$ capacitor (Fig. S3 in the Supporting Information). In all following results, $R$ is the resistance of the high-impedance $(100 \mathrm{M} \Omega)$ probe for the oscilloscope. $C_{f}$ and $C_{x l}$ can be calculated from measured voltage-time curves since the positive and negative voltage peaks correspond to the discharge process of $C_{f}$ and $C_{x I}$ respectively.

Fig. 2(a) presents measured output voltages of a $4 \mathrm{~cm} \times 4 \mathrm{~cm}$ sized TENG, with different maximum air gap $\left(x_{1}\right)$ values of $20.05 \mathrm{~mm}, 13.95 \mathrm{~mm}, 10.05 \mathrm{~mm}, 5 \mathrm{~mm}, 2 \mathrm{~mm}$, $1.05 \mathrm{~mm}$, and $0.55 \mathrm{~mm}$. At each $x_{1}$ value, output voltages were firstly measured for several working cycles with the switch keeping closed, and then measured while the switch was turned on and off in each working cycle as illustrated in Fig. 1(a). For a certain $x_{1}$ value, voltage peaks are stable among multiple cycles for each of these two measuring modes, meanwhile the voltage peaks under the switch on/off mode are higher than those under the switch close mode, because if the switch keeps closed all the time, charges can flow between the two electrodes not only at the two limit positions $\left(x=x_{1}\right.$ 
and $x=0$ ) but also during the movement of the counter electrode.

From measured voltages in Fig. 2(a), $C_{f}$ and $C_{x l}$ can be calculated according to equation (9). The voltage peaks measured under the switch controlling mode were used to do such calculations to get higher accuracy. Fig. 2(b)-(d) shows partially enlarged views of the output voltage with the $x_{1}$ set as $20.05 \mathrm{~mm}, 5 \mathrm{~mm}$, and $0.55 \mathrm{~mm}$ respectively. Fitting results in Fig. 2(b)-(d) give that calculated $C_{f}$ is $239 \mathrm{pF}, 241 \mathrm{pF}$, and $238 \mathrm{pF}$ for $x_{1}$ as $20.05 \mathrm{~mm}, 5 \mathrm{~mm}$, and $0.55 \mathrm{~mm}$ respectively, and corresponding $C_{x l}$ is $65 \mathrm{pF}, 67 \mathrm{pF}$, and $127 \mathrm{pF}$ respectively. For each $x_{1}$ value, three positive and negative voltage peaks were used to calculate $C_{f}$ and $C_{x l}$. Statistical results including the mean value and standard deviation of $C_{f}$ and $C_{x l}$ at each $x_{1}$ value are given in Fig. 3(a). With different $x_{1}$, $C_{f}$ basically keeps constant, which is in accordance with the variable capacitance model. For $C_{x l}$ formed by the air gap, it should increase with decreasing $x_{1}$ according to $C_{x l}=$ $S \varepsilon_{0} / x_{1}$, however, $C_{x l}$ only changes from $65 \mathrm{pF}$ to $67 \mathrm{pF}$ with $x_{1}$ from $20.05 \mathrm{~mm}$ to $5 \mathrm{~mm}$, while for $x_{1}$ as $2 \mathrm{~mm}, 1.05 \mathrm{~mm}$, and $0.55 \mathrm{~mm}, C_{x l}$ changes relatively apparently to $71 \mathrm{pF}$, $77 \mathrm{pF}$, and $125 \mathrm{pF}$. This signifies that there may be parasitic capacitance formed by air around the TENG or by electric components in the circuit [57], and when $x_{1}$ is large (e.g. larger than $2 \mathrm{~mm}$ ) the capacitance formed by the air gap between the counter electrode and the FEP film is rather smaller than the parasitic capacitance, so that $C_{x l}$ is dominated by parasitic capacitance. Only when $x_{1}$ is small, the influence of the air gap on $C_{x I}$ appears.

Fig. 3(b) shows the statistical results of positive $\left(V_{o c-f}\right)$ and negative $\left(V_{o c-x l}\right)$ voltage peak values in Fig. 2(a). For $x_{1}$ from $20.05 \mathrm{~mm}$ to $2 \mathrm{~mm}$, the mean value of $V_{o c-f}$ merely 
changes from $133 \mathrm{~V}$ to $129 \mathrm{~V}$, and the mean value of $V_{o c-x l}$ just decreases from $-377 \mathrm{~V}$ to $-358 \mathrm{~V}$. Only when $x_{1}$ decreases to $1.05 \mathrm{~mm}$ and $0.55 \mathrm{~mm}, V_{o c-f}$ slightly decrease to 122 $\mathrm{V}$ and $108 \mathrm{~V}$, and $V_{o c-x l}$ decreases to $-329 \mathrm{~V}$ and $-193 \mathrm{~V}$, respectively. These are in accordance with equations (5) and (7). According to equations (5) and (7), if $Q_{f}$ keeps constant, $V_{o c-f}$ is proportional to $\left(C_{f}+C_{x l}\right)^{-1}$, and $V_{o c-x l}$ is proportional to $C_{x I^{-1}}$. So that the absolute value of $V_{o c-x l}$ is always higher than $V_{o c-f}$ at each $x_{1}$ value. And since $C_{f}$ keeps constant but $C_{x l}$ increases with decreasing $x_{1}$, especially when $x_{1}$ is small as $1.05 \mathrm{~mm}$ and $0.55 \mathrm{~mm}$, both $V_{o c-f}$ and $V_{o c-x l}$ decrease with decreasing $x_{1}$.

Fig. 3(c) and 3(d) presents the $V_{o c-f}$ versus $\left(C_{f}+C_{x l}\right)^{-1}$, and $V_{o c-x l}$ versus $C_{x l^{-1}}$ respectively. Linear fitting results show that $V_{o c-f}$ is indeed proportional to $\left(C_{f}+C_{x l}\right)^{-1}$ with a high coefficient of determination as $0.945, V_{o c-x l}$ is proportional to $C_{x I^{-1}}$ with a high coefficient of determination as 0.98 , further verifying the validity of equations (5) and (7). The slopes of fitted lines in Fig. 3(c) and 3(d) represent the $Q_{f}$ value, which should be the same. However, the slope in Fig. 3(c) is 44414 pC, while that in Fig. 3(d) is $24454 \mathrm{pC}$. This can be explained by microscopic electric breakdown $[33,34]$. As we can see from Fig. 2(a), there is a small negative peak before each negative $V_{o c-x l}$ peak under the switch on/off mode. Fig. 3(e) presents the voltage signals measured under this mode with $x_{1}$ as $20.05 \mathrm{~mm}$ and the partially enlarged view of one small negative peak before one $V_{o c-x l}$ peak. This small negative peak means there is charge flowing through the oscilloscope, despite the switch was off when this peak appeared, implying that the button switch was temporally in failure. This might be caused by the high voltage generated when the counter electrode and the FEP surface with the respective charge of 
$Q_{f}$ and $-Q_{f}$ separated. At a certain distance, the high voltage between the counter and the back electrodes may exceed the normal operating voltage of the switch, resulting in some charge flowing between these two electrodes. Therefore, the residual charge on the counter electrode was less than actual $Q_{f}$ when $V_{o c-x l}$ peaks were measured. Therefore, to calculate $Q_{f}$, equation (5) was applied rather than equation (7). Calculated $Q_{f}$ with different $x_{1}$ is shown in Fig. 3(f), it basically keeps constant of $40 \mathrm{nC}$ during each working cycle and with different $x_{1}$, which is in good accordance with the capacitance model.

To further verify the capacitance model, short-circuit transferred charge amounts of the TENG with different $x_{1}$ were also measured as shown in Fig. 4(a). The total transferred charge from the state (i) to (iii) and from (iii) to (i) in Fig. 1 (a) should be $Q_{b}$ and $-Q_{b}$ respectively. Since $Q_{f}$ and $C_{f}$ keep constant with different $x_{1}$, and $C_{x l}$ hardly changes when $x_{1}$ is large but apparently increase when $x_{1}$ is small, $Q_{b}$ should hardly change when $x_{1}$ is large $(20 \mathrm{~mm}, 10 \mathrm{~mm}$, and $4.92 \mathrm{~mm})$ but apparently decrease when $x_{1}$ is small (1mm and $0.5 \mathrm{~mm}$ ) according to equation (3). Measured short-circuit transferred charges in Fig. 4(a) show exactly the variation trend as predicted by equation (3) and previous discussions.

Based on results and discussions above, the electric energy released by the TENG during each working cycle can be calculated according to equations (6) and (8). Fig. 4(b) presents the calculated $W_{f}$ and $W_{x l}$ and their sum with different $x_{1}$, they have a slight decline trend when $\mathrm{x}_{l}$ decrease from $20.05 \mathrm{~mm}$ to $2 \mathrm{~mm}$, but they decrease apparently when $\mathrm{x}_{1}$ decreases from $2 \mathrm{~mm}$ to $1.05 \mathrm{~mm}$ and $0.55 \mathrm{~mm}$. This means that the TENG 
could release more electric energy with a larger maximum air gap, but if the maximum air gap is larger than a specific value, parasitic capacitance dominates the $C_{x l}$ value, the influence of the maximum air gap on the harvested energy will be negligible. The sum of $W_{f}$ and $W_{x l}$ represents the possible maximum output energy of the TENG per working cycle, which excludes the influence of input mechanical energy power and the power management unit on the performance of the TENG. That means the sum of $W_{f}$ and $W_{x I}$ could be utilized as a criterion to compare the property of TENGs with different structure and power management system, in diverse working conditions, which is quite important on the way of developing high-performance TENG.

\subsection{Improving the performance}

The variable capacitance model discussed above is built based on the switch on/off working mode which makes the TENG output maximum power under the same condition $[58,59,64,65]$. Though the TENG normally works without switch control, this model is instructive in improving the output power of TENG. According to equation (5), (6), (7), and (8), the output power of the TENG under the same working condition can be improved through two different routes, one is to increase the charge amount on the FEP film $\left(Q_{f}\right)$ and the other is to optimize the capacitance values $\left(C_{f}\right.$ and $\left.C_{x l}\right)$. For the TENG with a certain lateral dimension, to optimize the capacitance values, dielectric constant and thickness of the electret film, the maximum air gap $\left(x_{1}\right)$ and the parasitic capacitance in circuits, these parameters should be considered, which concern the choice of materials and structure designs [31]. While for a TENG with a certain material, structure and a given working condition, $C_{f}$ and $C_{x l}$ values hardly change, maximum open-circuit 
voltages $\left(V_{o c-f}\right.$ and $\left.V_{o c-x l}\right)$ are proportional to $\mathrm{Q}_{f}$, and the maximum output energy ( $W_{f}$ and $W_{x}$ ) is proportional to $\mathrm{Q}_{f}^{2}$ according to our model, which are in accordance with other researchers' models $[33-35,58,60]$.

In this study, to improve the output voltage and power of the TENG, we increase $\mathrm{Q}_{f}$ firstly by using an adhesive tape-peeling method. Fig. 5(a) illustrates the process and principle of this method. Briefly, one piece of adhesive tape was firstly pasted on the FEP film, then triboelectrification occurs when the tape was peeled off, making the acrylic adhesive on the sticky side of tape positively charged, and the FEP film negatively charged. Due to the strong adhesive force between the tape and the FEP film, and the wide gap between acrylic and FEP in the triboelectric series $[31,66]$, the triboelectrification is so strong that can build up high electric field between the two polymer film, and can even generate X-ray if the tape is peeled off in low air pressure [67]. Possible air ionization during this process may also contribute to the charge injection.

The effectiveness of this charging method can be verified by measuring the surface potential of the FEP film which is proportional to the average charge density on the FEP film [32]. Fig. 5(b) shows the surface potential of the $4 \times 4 \mathrm{~cm}^{2}$ FEP film with increasing counts of such treatment, before and after the linear motor test. The surface potentials are all negative even without treatment, meaning that the FEP film was negatively charged. With increasing treatment counts, the surface potential was monotonously improved from $-360 \mathrm{~V}$ to $-2850 \mathrm{~V}$. And the surface potential almost kept unchanged before and after the linear motor test, showing the temporal stability of 
negative charges on the FEP film. It should be noted that only one tape-peeling process was performed during each except for the fifth treatment. During the fifth treatment, the surface potential is more difficult to be improved, three paste and peel-off processes were performed. And it can be noticed that a little change in surface potential occurs after linear motor test for the FEP film after the fifth treatment. We assume that the charge amount on the FEP film might approach saturation after the fourth treatment, so that negative charges become more difficult to be deposited and less stable if deposited on the FEP film.

The improvement in the output voltage and current of the $4 \times 4 \mathrm{~cm}^{2}$ TENG by this treatment method for the FEP film is presented in Fig. 6. All results were measured with $x_{l}$ set as $20.05 \mathrm{~mm}$, without switch control in the circuit. With increasing treatment count, the output voltage increases monotonously as shown in Fig. 6(a). Statistical results of the voltage peak values with increasing FEP film surface potentials controlled by the tape-peeling treatment are presented in Fig. 6(b), where the first and last three voltage peaks from each test result in Fig. 6(a) are used for the statistic. The mean positive peak value increases from $41 \mathrm{~V}$ to $195 \mathrm{~V}$, and negative peak value increases from $-83 \mathrm{~V}$ to $-363 \mathrm{~V}$. The output current density also increases monotonously with increasing treatment counts as shown in Fig. 6(c). Measured current signals are not so stable as voltages. This is because the resistance of the external circuit was low when the current was measured so that the current decayed fast. While the data sampling rate of the current measuring system is not so high, only one data point can be acquired in one current peak (shown in Fig. S4, Supporting Information), therefore the maximum current 
value in one current peak may not always be recorded. Nevertheless, an increasing trend of the current density with treatment count can still be recognized from Fig. 6(c). Five highest positive and negative values from each test result in Fig. 6(c) were used to do statistical analysis. Mean positive peak current density was improved from $1.5 \mathrm{~mA} / \mathrm{m}^{2}$ to $7.3 \mathrm{~mA} / \mathrm{m}^{2}$, and negative peak current density improved $-1.3 \mathrm{~mA} / \mathrm{m}^{2}$ to $-5.3 \mathrm{~mA} / \mathrm{m}^{2}$ as shown in Fig. 6(d).

The improvement in the short-circuit transferred charge amount was also measured from another $4 \mathrm{~cm} \times 4 \mathrm{~cm}$ TENG and shown in Fig. 7(a), keeping all measuring conditions unchanged. After three times of treatment, the transferred charge amount in each half working cycle was improved from $\sim 19 \mathrm{nC}$ to $\sim 53 \mathrm{nC}$. The corresponding output voltages of the TENG during this transferred charge test were also measured and given in Fig. S5 in the Supporting Information. With these transferred charge amounts together with the output voltages, the triboelectric charge density and the maximum instantaneous power density of this TENG can be calculated and compared with results in some references that have established an evaluating standard and a theoretical model for TENGs [58, 60]. To do such a calculation and comparison, some theoretical and experimental details need to be discussed, which can be found in Table S1, Supplementary Note 2, and Table S2 and Supplementary Note 3 in the Supporting Information. After three times of treatment, the effective triboelectric charge density was increased from $\sim 12 \mu \mathrm{C} / \mathrm{m}^{2}$ to $\sim 33 \mu \mathrm{C} / \mathrm{m}^{2}$, and the corresponding maximum instantaneous power density for the contact and separate process was increased from $\sim 29 \mathrm{~mW} / \mathrm{m}^{2}$ and $\sim 92 \mathrm{~mW} / \mathrm{m}^{2}$ to $\sim 515 \mathrm{~mW} / \mathrm{m}^{2}$ and $833 \mathrm{~mW} / \mathrm{m}^{2}$, respectively. Fig. 7(b) presents the 
transferred charge amount durability measurement for more than 1200 working cycles of the TENG after treatments, with the $x_{1}$ set as $2 \mathrm{~mm}$ to measure more working cycles during the test time. The transferred charge amount has no obvious change, suggesting that the reserved charge on the FEP film is stable, and the FEP film becomes a piece of electret. Figure 7(c) shows the output voltage of the TENG during about 1000 working cycles, with the $x_{1}$ set as $5 \mathrm{~mm}$ and the period for each working cycle as $0.5 \mathrm{~s}$. The output voltage is also very stable because of the charge stability on the FEP film. These results demonstrate the effectiveness and durability of improving the output of the TENG by the tape-peeling process. In comparison with widely used thermal charging or corona charging method, though this method is not so accurate in controlling the surface potential of the electret to a specific desired value, its facility and effectiveness make it competitive.

\subsection{Energy harvesting demonstrations}

Basic characteristics of the TENG have been studied with linear motor-driven tests as presented above. To be more practical, the TENG should be able to harvest energy from natural motions in the environment. Since the $100 \mu \mathrm{m}$-thick FEP film is very flexible, it can be driven by wind to flap or vibrate thus to change the air-gap capacitance, making the FEP film-based TENG be able to generate electricity from the wind. Fig. 8(a) shows the photograph of the wind-driven TENG with an $8 \mathrm{~cm} \times 8 \mathrm{~cm}$ sized FEP film, and the diagram illustrating its working and measuring process. The wind comes from a hairdryer, with a wind speed of $\sim 18 \mathrm{~m} / \mathrm{s}$, and blows through a slit of $\sim 3 \mathrm{~mm}$ between the FEP film and the Al foil. The FEP film with one corner fixed vibrates under the wind 
blowing, repeatedly flaps and separates from the Al foil, changing the capacitance between the FEP film and the Al foil. The change of this capacitance generates a potential difference between the back electrode on the FEP film and the Al foil as discussed in section 3.1, and this change is obvious especially when the FEP film and the Al foil have a relative motion near the contact status as discussed in section 3.2. Such flapping of the FEP film is competent to generate enough potential difference which can be detected by the oscilloscope.

Fig. 8(b) gives output voltages of the $8 \mathrm{~cm} \times 8 \mathrm{~cm}$ sized FEP film-based TENG under wind blowing. The four data parts (i), (ii), (iii), and (iv) shown in Fig. 8(b) were measured in sequence. Among them, (i) is the output of the TENG without any treatment to the FEP film surface, (ii) is the output after the TENG kept working under wind blowing for 30 s to charge a commercial $10 \mu \mathrm{F}$ capacitor (diagram of electric circuit given in Fig. 8(d)), (iii) was measured after the FEP film was treated twice by the tape-peeling process discussed in section 3.3, and (iv) was measured after the TENG worked under wind blowing for another 30 s to charge the $10 \mu \mathrm{F}$ capacitor again. For each part, the output voltage obviously appeared when the wind began and disappeared when the wind stopped, and because the vibration of the FEP film was irregular, the measured voltage peak value is also irregular. From (i) to (iv), the output voltage has a gradually increasing trend, which means that the charge amount on the FEP surface can be improved not only by the tape-peeling treatment but also during the wind blowing process. A possible explanation is that microscopic relative sliding occurs between the FEP film and the Al foil when the FEP film flutters fiercely and irregularly under wind blowing, the friction 
force during this sliding process promotes the triboelectrification between the FEP and the Al foil surfaces, increasing the charge amount on both surfaces. The surface potential of the FEP film before each test from (i) to (iv) was measured and presented in Fig. 8(c). The gradual increase of the surface potential from (i) to (iv) verifies the increase of the charge amount.

Fig. $8(\mathrm{~d})$ is the circuit diagram of the TENG driven by wind to charge a capacitor $\left(C_{s}\right)$ for energy storage, or to power electric loads. A rectifier bridge was assembled in the circuit to convert generated irregular $\mathrm{AC}$ voltage output to $\mathrm{DC}$ output, which is essential for charging $C_{s}$. When the two switches in the circuit are both open, the oscilloscope detects the rectified voltage output of the TENG under wind blowing. When the switch connected with $\mathrm{C}_{\mathrm{s}}$ is closed but the other one is open, the oscilloscope measures the charging curve of $\mathrm{C}_{\mathrm{s}}$, in the contrary switch condition, the oscilloscope will measure the partial voltage across electric loads. Fig. 8(e) gives the no-rectified and rectified output voltages of the TENG under wind blowing. There will be some energy loss during the rectification, which might be caused by the reverse current leakage of the diodes in the rectifier bridge. The rectification result of a sinusoidal wave by the rectifier bridge is given in Fig. S6 in the Supporting Information to show the voltage drop caused by rectifying.

In Fig. 8(f), the two charging curves of a $10 \mu \mathrm{F} \mathrm{C}_{\mathrm{s}}$ during the 30 s working processes of the wind-driven TENG between test (i) and (ii), and between test (iii) and (iv) are presented. Before tape treatment, $\mathrm{C}_{\mathrm{s}}$ was charged from $0 \mathrm{~V}$ to a voltage of $3.9 \mathrm{~V}$ in $30 \mathrm{~s}$ ((i) to (ii)), corresponding to an average charging power of $\sim 2.5 \mu \mathrm{W}$; after the FEP film 
being treated with tape, $\mathrm{C}_{\mathrm{s}}$ was charged from $0 \mathrm{~V}$ to $10.9 \mathrm{~V}$ in $30 \mathrm{~s}$ ((iii) to (iv)), corresponding to an improved average charging power of $\sim 19.8 \mu \mathrm{W}$ which is enough to drive a sensor unit or other low power dissipation electronics $[8,68]$. It is worthy to be noted that these average charging powers are calculated based on the energy stored in $\mathrm{C}_{\mathrm{s}}$. The high-impedance probe of the oscilloscope and the rectifier bridge also consume some power, so the actual average output power of the TENG is higher than these $\mathrm{C}_{\mathrm{s}}$ charging powers. And the maximum instantaneous power output of the TENG, which is calculated based on the peak voltage and current value and the Ohm's law in other works [69-72], is much higher. Other factors such as the wind speed and incident angle [73, 74], and the impedance match between the external load and the TENG $[61,75,76]$, those influence the output of this type of TENG have been studied by other researchers, which will not be discussed here.

This film-based TENG not only can harvest energy from wind but is also capable of harvesting energy from low-frequency human motions. In the photograph Fig. 8(g), 42 LEDs were directly lighted up by the TENG with a rectifier bridge when the FEP film was slightly and slowly flapped by a human hand. The video for this process is provided as Video S1.

\section{Conclusions}

In summary, we proposed a variable capacitance model for the FEP electret film-based TENG. The validity of this model was verified with capacitance values calculated from output voltages of a $4 \times 4 \mathrm{~cm}^{2}$ TENG under linear motor test. The calculation is based on the capacitor discharge curve fitting method, which is applied to 
TENG for the first time. This model provides a criterion for evaluating the possible maximum output energy of the TENG during per working cycle, and it reveals that improving charge amount on electrets and optimizing capacitance values are two technic routes to improve the output of TENG. We also originally used drastic triboelectrification generated by an adhesive tape-peeling method to charge the FEP electret film. The surface potential of the $4 \times 4 \mathrm{~cm}^{2}$ FEP film was thus improved from about $-360 \mathrm{~V}$ to about $-2850 \mathrm{~V}$, the positive voltage peak of the TENG was improved from $41 \mathrm{~V}$ to $195 \mathrm{~V}$, negative peak improved from $-83 \mathrm{~V}$ to $-363 \mathrm{~V}$, positive peak current density improved from $1.5 \mathrm{~mA} / \mathrm{m}^{2}$ to $7.3 \mathrm{~mA} / \mathrm{m}^{2}$, and negative peak current density improved from -1.3 $\mathrm{mA} / \mathrm{m}^{2}$ to $-5.3 \mathrm{~mA} / \mathrm{m}^{2}$. This facile and effective charging method might be inspiring for electret preparation. Besides, a TENG made with an $8 \times 8 \mathrm{~cm}^{2}$ FEP film was driven by wind to charge a $10 \mu \mathrm{F}$ capacitor for energy conversion and storage. Before the tape-peeling treatment, the wind-driven TENG can charge the $10 \mu \mathrm{F}$ capacitor from 0 to $3.9 \mathrm{~V}$ in $30 \mathrm{~s}$, corresponding to an average charging power of $\sim 2.5 \mu \mathrm{W}$; after the tape-peeling treatment, the same capacitor was charged from $0 \mathrm{~V}$ to $10.9 \mathrm{~V}$ in $30 \mathrm{~s}$, corresponding to an improved average charging power of $\sim 19.8 \mu \mathrm{W}$. Additionally, we demonstrated that this TENG can harvest energy from low-frequency human motions. When the FEP film was fluttered slightly by a human hand, the TENG directly lighted up 42 LEDs. This miniature TENG prototype shows its potential applications in self-powered systems that obtain energy from irregular mechanical motions in the surrounding environment, which deserves further researches.

\section{Acknowledgements}


This work is financially supported by the China Scholarship Council (CSC). The authors also give thanks to Mr. Éric Perrin for his help in setting up the linear motor systems.

\section{References}

[1] Z.L. Wang, J. Chen, L. Lin, Progress in triboelectric nanogenerators as a new energy technology and self-powered sensors, Energy Environ. Sci., 8 (2015) 2250-2282.

[2] Z.L. Wang, L. Lin, J. Chen, S. Niu, Y. Zi, Triboelectric Nanogenerators, Springer, 2016.

[3] R. Pan, W. Xuan, J. Chen, S. Dong, H. Jin, X. Wang, H. Li, J. Luo, Fully biodegradable triboelectric nanogenerators based on electrospun polylactic acid and nanostructured gelatin films, Nano Energy, 45 (2018) 193-202.

[4] Z.L. Wang, Catch wave power in floating nets, Nature, 542 (2017) 159-160.

[5] J. Zhong, Q. Zhong, G. Chen, B. Hu, S. Zhao, X. Li, N. Wu, W. Li, H. Yu, J. Zhou, Surface charge self-recovering electret film for wearable energy conversion in a harsh environment, Energy Environ. Sci., 9 (2016) 3085-3091.

[6] S.P. Beeby, M.J. Tudor, N.M. White, Energy harvesting vibration sources for microsystems applications, Meas. Sci. Technol., 17 (2006) R175-R195.

[7] Z.L. Wang, On Maxwell's displacement current for energy and sensors: the origin of nanogenerators, Mater. Today, 20 (2017) 74-82.

[8] P.D. Mitcheson, E.M. Yeatman, G.K. Rao, A.S. Holmes, T.C. Green, Energy harvesting from human and machine motion for wireless electronic devices, Proc. IEEE, 96 (2008) 1457-1486. 
[9] B. Yang, C. Lee, W. Xiang, J. Xie, J.H. He, R.K. Kotlanka, S.P. Low, H. Feng, Electromagnetic energy harvesting from vibrations of multiple frequencies, J. Micromech. Microeng., 19 (2009) 035001.

[10] Z.L. Wang, J. Song, Piezoelectric nanogenerators based on zinc oxide nanowire arrays, Science, 312 (2006) 242-246.

[11] X. Wang, J. Song, J. Liu, Z.L. Wang, Direct-current nanogenerator driven by ultrasonic waves, Science, 316 (2007) 102-105.

[12] S. Xu, B.J. Hansen, Z.L. Wang, Piezoelectric-nanowire-enabled power source for driving wireless microelectronics, Nat. Commun., 1 (2010) 93.

[13] J. Dicken, P.D. Mitcheson, I. Stoianov, E.M. Yeatman, Power-extraction circuits for piezoelectric energy harvesters in miniature and low-power applications, IEEE Trans. Power Electron., 27 (2012) 4514-4529.

[14] P.D. Mitcheson, P. Miao, B.H. Stark, E.M. Yeatman, A.S. Holmes, T.C. Green, MEMS electrostatic micropower generator for low frequency operation, Sens. Actuators, A, 115 (2004) 523-529.

[15] P. Miao, A. Holmes, E. Yeatman, T. Green, P. Mitcheson, Electrostatics 2003, CRC Press, Edinburgh, 2004.

[16] Y. Naruse, N. Matsubara, K. Mabuchi, M. Izumi, S. Suzuki, Electrostatic micro power generation from low-frequency vibration such as human motion, J. Micromech. Microeng., 19 (2009) 094002.

[17] T.G. McKay, B.M. O’Brien, E.P. Calius, I.A. Anderson, Soft generators using dielectric elastomers, Appl. Phys. Lett., 98 (2011) 142903. 
[18] C. Jean-Mistral, T. Vu-Cong, A. Sylvestre, On the power management and electret hybridization of dielectric elastomer generators, Smart Mater. Struct., 22 (2013) 104017. [19] F.R. Fan, Z.Q. Tian, Z.L. Wang, Flexible triboelectric generator!, Nano Energy, 1 (2012) 328-334.

[20] Z.L. Wang, Triboelectric nanogenerators as new energy technology and self-powered sensors-Principles, problems and perspectives, Faraday Discuss, 176 (2015) 447-458.

[21] S.H. Wang, L. Lin, Z.L. Wang, Nanoscale triboelectric-effect-enabled energy conversion for sustainably powering portable electronics, Nano Lett., 12 (2012) 6339-6346.

[22] J. Zhong, Q. Zhong, F. Fan, Y. Zhang, S. Wang, B. Hu, Z.L. Wang, J. Zhou, Finger typing driven triboelectric nanogenerator and its use for instantaneously lighting up LEDs, Nano Energy, 2 (2013) 491-497.

[23] S.W. Chen, C.Z. Gao, W. Tang, H.R. Zhu, Y. Han, Q.W. Jiang, T. Li, X. Cao, Z.L. Wang, Self-powered cleaning of air pollution by wind driven triboelectric nanogenerator, Nano Energy, 14 (2015) 217-225.

[24] S.B. Jeon, S. Kim, S.J. Park, M.L. Seol, D. Kim, Y.K. Chang, Y.K. Choi, Self-powered electro-coagulation system driven by a wind energy harvesting triboelectric nanogenerator for decentralized water treatment, Nano Energy, 28 (2016) 288-295.

[25] G. Zhu, J. Chen, T. Zhang, Q. Jing, Z.L. Wang, Radial-arrayed rotary electrification for high performance triboelectric generator, Nat. Commun., 5 (2014) 3426.

[26] X. Li, J. Tao, J. Zhu, C. Pan, A nanowire based triboelectric nanogenerator for 
harvesting water wave energy and its applications, APL Mater., 5 (2017) 074104.

[27] Y. Yang, G. Zhu, H.L. Zhang, J. Chen, X.D. Zhong, Z.H. Lin, Y.J. Su, P. Bai, X.N. Wen, Z.L. Wang, Triboelectric nanogenerator for harvesting wind energy and as self-powered wind vector sensor system, ACS Nano, 7 (2013) 9461-9468.

[28] X. Wang, S. Niu, F. Yi, Y. Yin, C. Hao, K. Dai, Y. Zhang, Z. You, Z.L. Wang, Harvesting ambient vibration energy over a wide frequency range for self-powered Electronics, ACS Nano, 11 (2017) 1728-1735.

[29] B. Meng, W. Tang, Z.H. Too, X.S. Zhang, M.D. Han, W. Liu, H.X. Zhang, A transparent single-friction-surface triboelectric generator and self-powered touch sensor, Energy Environ. Sci., 6 (2013) 3235-3240.

[30] J. Zhao, H. Li, C. Li, Q. Zhang, J. Sun, X. Wang, J. Guo, L. Xie, J. Xie, B. He, Z. Zhou, C. Lu, W. Lu, G. Zhu, Y. Yao, MOF for template-directed growth of well-oriented nanowire hybrid arrays on carbon nanotube fibers for wearable electronics integrated with triboelectric nanogenerators, Nano Energy, 43(2018) 420-431.

[31] Z.L. Wang, Triboelectric nanogenerators as new energy technology for self-powered systems and as active mechanical and chemical sensors, ACS Nano, 7 (2013) 9533-9557.

[32] B. Gross, G.M. Sessler, Electrets, Springer-Verlag Berlin Heidelberg, 1987.

[33] J. Wang, C. Wu, Y. Dai, Z. Zhao, A. Wang, T. Zhang, Z.L. Wang, Achieving ultrahigh triboelectric charge density for efficient energy harvesting, Nat. Commun., 8 (2017) 88 .

[34] S. Wang, Y. Xie, S. Niu, L. Lin, C. Liu, Y.S. Zhou, Z.L. Wang, Maximum surface charge density for triboelectric nanogenerators achieved by ionized - air injection: 
methodology and theoretical understanding, Adv. Mater., 26 (2014) 6720-6728.

[35] X.Y. Wei, G. Zhu, Z.L. Wang, Surface-charge engineering for high-performance triboelectric nanogenerator based on identical electrification materials, Nano Energy, 10 (2014) 83-89.

[36] R.D.I.G. Dharmasena, K.D.G.I. Jayawardena, C.A. Mills, J.H.B. Deane, J.V. Anguita, R.A. Dorey, S.R.P. Silva, Triboelectric nanogenerators: providing a fundamental framework, Energy Environ. Sci., 10 (2017) 1801-1811.

[37] H.Y. Li, L. Su, S.Y. Kuang, C.F. Pan, G. Zhu, Z.L. Wang, Significant enhancement of triboelectric charge density by fluorinated surface modification in nanoscale for converting mechanical energy, Adv. Funct. Mater., 25 (2015) 5691-5697.

[38] G.M. Sessler, J. Hillenbrand, Electromechanical response of cellular electret films, Appl. Phys. Lett., 75 (1999) 3405-3407.

[39] B. Gross, G.M. Sessler, J.E. West, Charge dynamics for electron - irradiated polymer - foil electrets, J. Appl. Phys., 45 (1974) 2841-2851.

[40] S. Bauer, R. Gerhard-Multhaupt, G.M. Sessler, Ferroelectrets: Soft electroactive foams for transducers, Phys. Today, 57 (2004) 37-43.

[41] Y. Suzuki, D. Miki, M. Edamoto, M. Honzumi, A MEMS electret generator with electrostatic levitation for vibration-driven energy-harvesting applications, J. Micromech. Microeng., 20 (2010) 104002.

[42] Z. Yang, J. Wang, J. Zhang, A high-performance micro electret power generator based on microball bearings, J. Micromech. Microeng., 21 (2011) 065001.

[43] J. Hillenbrand, N. Behrendt, V. Altstädt, H.W. Schmidt, G.M. Sessler, Electret 
properties of biaxially stretched polypropylene films containing various additives, J. Phys. D: Appl. Phys., 39 (2006) 535-540.

[44] Z. Xia, R. Gerhard-Multhaupt, W. Künstler, A. Wedel, R. Danz, High surface-charge stability of porous polytetrafluoroethylene electret films at room and elevated temperatures, J. Phys. D: Appl. Phys., 32 (1999) L83-L85.

[45] C. Qiang, PTFE electret negative charge stability after RF plasma treatment, J. Phys. D: Appl. Phys., 35 (2002) 2939-2944.

[46] B. Wang, J. Zhong, Q. Zhong, N. Wu, X. Cheng, W. Li, K. Liu, L. Huang, B. Hu, J. Zhou, Sandwiched composite fluorocarbon film for flexible electret generator, Adv. Electron. Mater., 2 (2016) 1500408.

[47] T. Sterken, P. Fiorini, G. Altena, C. Van Hoof, R. Puers, Proc. 14th Int. Conf. Solid-State Sensors, Actuators, and Microsystems, IEEE, New York, 2007.

[48] Y. Arakawa, Y. Suzuki, N. Kasagi, Micro seismic power generator using electret polymer film, Proc. PowerMEMS, (2004) 187-190.

[49] M. Suzuki, T. Takahashi, S. Aoyagi, Development of a high-performance fluorpolymer electret mixed with nano-particles and tts application to vibration energy harvesting, J. Phys.: Conf. Ser., 557 (2014) 012062.

[50] H.M. Xiao, G.J. Chen, X.M. Chen, Z. Chen, A flexible electret membrane with persistent electrostatic effect and fesistance to harsh environment for energy harvesting, Sci. Rep., 7 (2017) 8443.

[51] G.J. Chen, Y.F. Li, H.M. Xiao, X. Zhu, A micro-oscillation-driven energy harvester based on a flexible bipolar electret membrane with high output power, J. Mater. Chem. A, 
5 (2017) 4150-4155.

[52] X. Zhang, G.M. Sessler, Y. Xue, X. Ma, Audio and ultrasonic responses of laminated fluoroethylenepropylene and porous polytetrafluoroethylene films with different charge distributions, J. Phys. D: Appl. Phys., 49 (2016) 205502.

[53] H.T. Baytekin, A.Z. Patashinski, M. Branicki, B. Baytekin, S. Soh, B.A. Grzybowski, The mosaic of surface charge in contact electrification, Science, 333 (2011) 308-312.

[54] R. Budakian, K. Weninger, R.A. Hiller, S.J. Putterman, Picosecond discharges and stick-slip friction at a moving meniscus of mercury on glass, Nature, 391 (1998) 266-268.

[55] T. Zhou, L. Zhang, F. Xue, W. Tang, C. Zhang, Z.L. Wang, Multilayered electret films based triboelectric nanogenerator, Nano Res., 9 (2016) 1442-1451.

[56] Y. Yang, H. Zhang, X. Zhong, F. Yi, R. Yu, Y. Zhang, Z.L. Wang, Electret film-enhanced triboelectric nanogenerator matrix for self-powered instantaneous tactile imaging, ACS Appl. Mater. Interfaces, 6 (2014) 3680-3688.

[57] B.H. Stark, P.D. Mitcheson, P. Miao, T.C. Green, E.M. Yeatman, A.S. Holmes, Converter circuit design, semiconductor device selection and analysis of parasitics for micropower electrostatic generators, IEEE Trans. Power Electron., 21 (2006) 27-37.

[58] Y. Zi, S. Niu, J. Wang, Z. Wen, W. Tang, Z.L. Wang, Standards and figure-of-merits for quantifying the performance of triboelectric nanogenerators, Nat. Commun., 6 (2015) 8376.

[59] P.D. Mitcheson, T. Sterken, C. He, M. Kiziroglou, E.M. Yeatman, R. Puers, 
Electrostatic microgenerators, Meas. Control, 41 (2008) 114-119.

[60] S. Niu, S. Wang, L. Lin, Y. Liu, Y.S. Zhou, Y. Hu, Z.L. Wang, Theoretical study of contact-mode triboelectric nanogenerators as an effective power source, Energy Environ. Sci., 6 (2013) 3576-3583.

[61] S. Niu, Z.L. Wang, Theoretical systems of triboelectric nanogenerators, Nano Energy, 14 (2015) 161-192.

[62] S. Boisseau, G. Despesse, T. Ricart, E. Defay, A. Sylvestre, Cantilever-based electret energy harvesters, Smart Mater. Struct., 20 (2011) 105013.

[63] Y. Suzuki, Micro-nano mechatronics and human science, 2008. MHS 2008. International Symposium on, IEEE2008, pp. 180-183.

[64] P.D. Mitcheson, T.C. Green, Maximum effectiveness of electrostatic energy harvesters when coupled to interface circuits, IEEE Trans. Circuits Syst. I-Regul. Pap., 59 (2012) 3098-3111.

[65] Y. Zi, J. Wang, S. Wang, S. Li, Z. Wen, H. Guo, Z.L. Wang, Effective energy storage from a triboelectric nanogenerator, Nat. Commun., 7 (2016) 10987.

[66] A.F. Diaz, R.M. Felix-Navarro, A semi-quantitative tribo-electric series for polymeric materials: the influence of chemical structure and properties, J. Electrost., 62 (2004) 277-290.

[67] C.G. Camara, J.V. Escobar, J.R. Hird, S.J. Putterman, Correlation between nanosecond X-ray flashes and stick-slip friction in peeling tape, Nature, 455 (2008) 1089-1092.

[68] K.-E. Byun, M.-H. Lee, Y. Cho, S.-G. Nam, H.-J. Shin, S. Park, Potential role of 
motion for enhancing maximum output energy of triboelectric nanogenerator, APL Mater., 5 (2017) 074107.

[69] Y.N. Xie, S.H. Wang, L. Lin, Q.S. Jing, Z.H. Lin, S.M. Niu, Z.Y. Wu, Z.L. Wang, Rotary triboelectric nanogenerator based on a hybridized mechanism for harvesting wind energy, ACS Nano, 7 (2013) 7119-7125.

[70] L.B. Huang, W. Xu, G.X. Bai, M.C. Wong, Z.B. Yang, J.H. Hao, Wind energy and blue energy harvesting based on magnetic-assisted noncontact triboelectric nanogenerator, Nano Energy, 30 (2016) 36-42.

[71] C. Han, C. Zhang, W. Tang, X. Li, Z.L. Wang, High power triboelectric nanogenerator based on printed circuit board (PCB) technology, Nano Res., 8 (2015) 722-730.

[72] Q. Zhong, J. Zhong, B. Hu, Q. Hu, J. Zhou, Z.L. Wang, A paper-based nanogenerator as a power source and active sensor, Energy Environ. Sci., 6 (2013) 1779-1784.

[73] M.L. Seol, J.H. Woo, S.B. Jeon, D. Kim, S.J. Park, J. Hur, Y.K. Choi, Vertically stacked thin triboelectric nanogenerator for wind energy harvesting, Nano Energy, 14 (2015) 201-208.

[74] H. Yong, J. Chung, D. Choi, D. Jung, M. Cho, S. Lee, Highly reliable wind-rolling triboelectric nanogenerator operating in a wide wind speed range, Sci. Rep., 6 (2016) 33977.

[75] S. Niu, Y. Liu, Y.S. Zhou, S. Wang, L. Lin, Z.L. Wang, Optimization of triboelectric nanogenerator charging systems for efficient energy harvesting and storage, 
IEEE Trans. Electron Devices, 62 (2015) 641-647.

[76] F. Xi, Y. Pang, W. Li, T. Jiang, L. Zhang, T. Guo, G. Liu, C. Zhang, Z.L. Wang, Universal power management strategy for triboelectric nanogenerator, Nano Energy, 37 (2017) 168-176. 

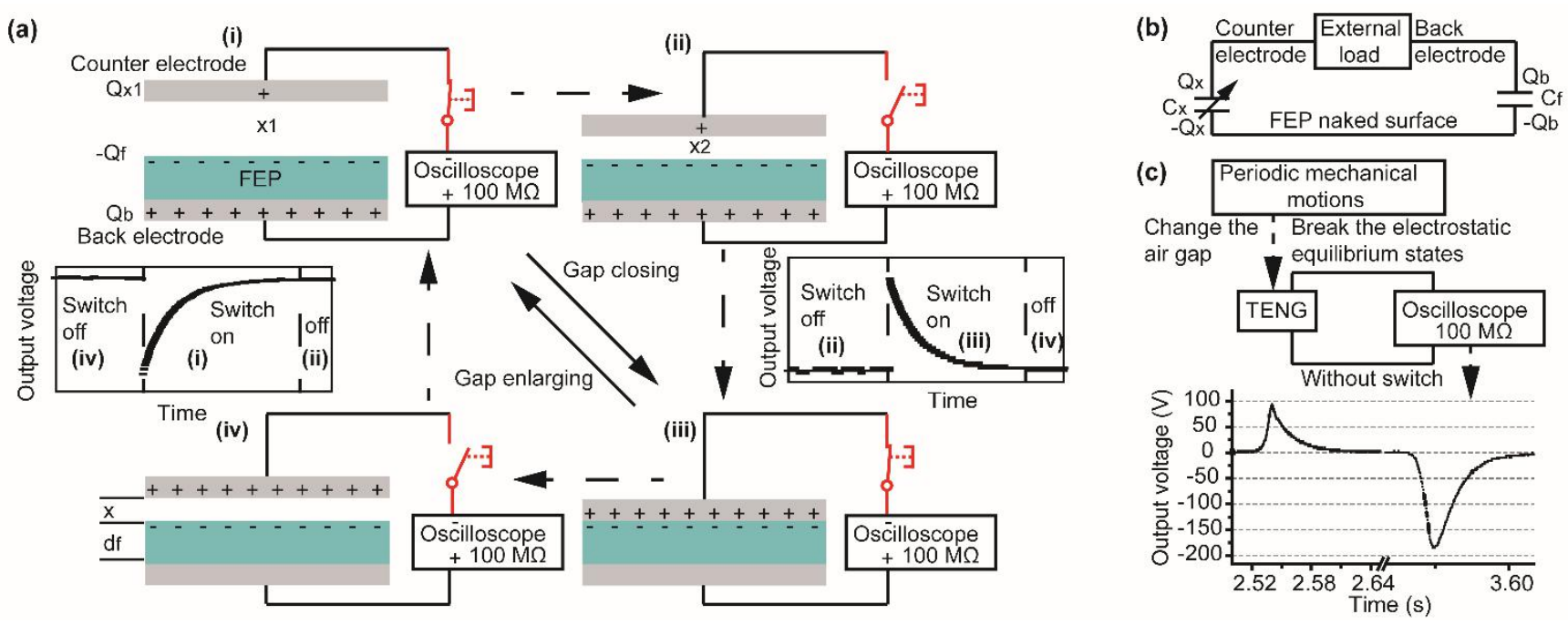

Fig. 1. Working principle of the TENG explained with the variable capacitance model. (a) The schematic of one working cycle of the FEP electret film-based gap varying TENG. (b) The equivalent circuit diagram of the capacitance model of the TENG. (c) The diagram showing the working process of the TENG and its output voltage without switch in the external circuit. 

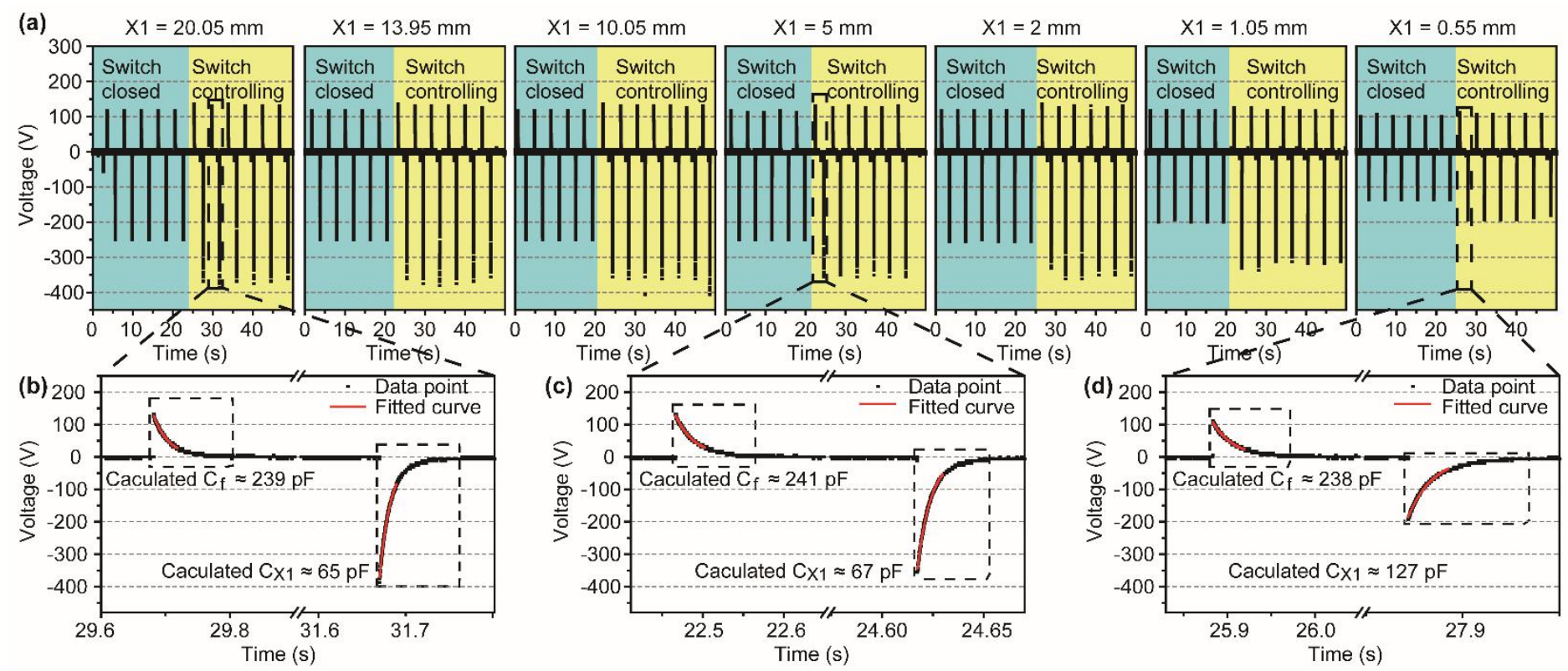

Fig. 2. The capacitor discharge fitting method for verifying the variable capacitance model. (a)

The output voltages of a $4 \times 4 \mathrm{~cm} 2$ sized TENG with different maximum air gap $\mathrm{x} 1$ values, from left to right, $\mathrm{x} 1$ varies from $20.05 \mathrm{~mm}$ to $0.55 \mathrm{~mm}$. (b)-(d) Partially enlarged views of the output voltage peaks with $\mathrm{x} 1$ set as $20.05 \mathrm{~mm}, 5 \mathrm{~mm}$, and $0.55 \mathrm{~mm}$ respectively, and corresponding fitting results giving calculated $\mathrm{Cf}$ and $\mathrm{Cx} 1$ values. 
(a)

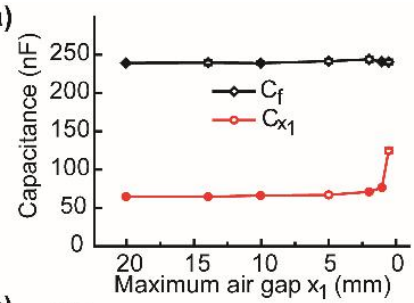

(c)

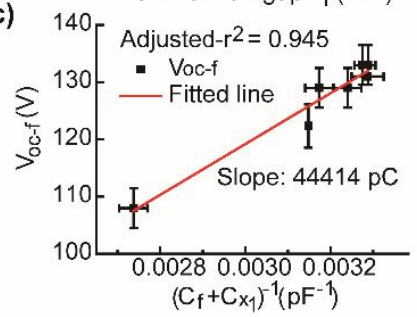

(e)

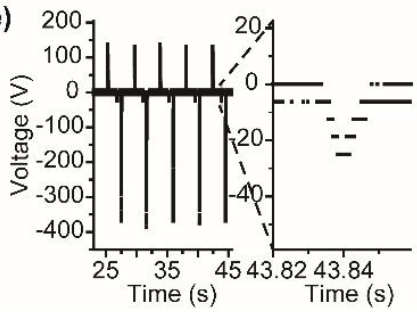

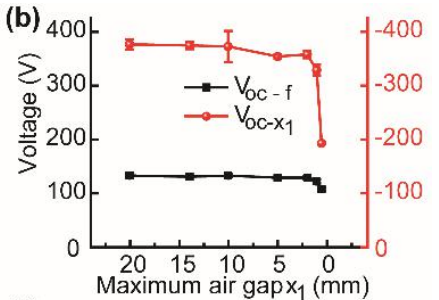

(d)
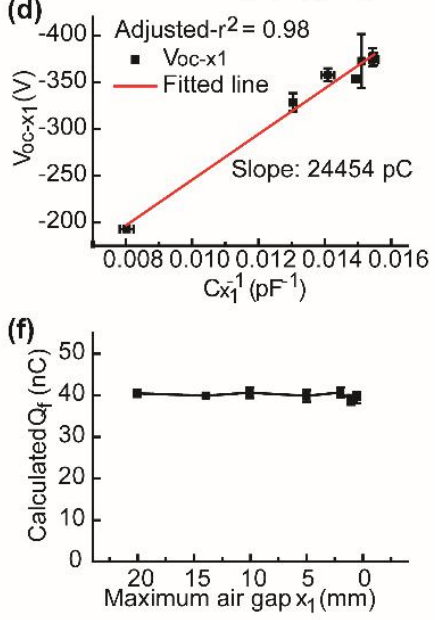

Fig. 3. Verification of the variable capacitance model. (a) Statistic results of calculated $C_{f}$ and $C_{x l}$ values with different $x_{1}$. (b) Statistic results of calculated peak $V_{o c-f}$ and $V_{o c-x l}$ with different $x_{1}$. (c) $V_{o c-f}$ as a function of $\left(C_{f}+C_{x l}\right)^{-1}$. (d) $V_{o c-x l}$ as a function of $C_{x l}{ }^{-1}$. (e) Partially enlarged view of the output voltage shows the electric leakage before $V_{o c-x l}$ peaks. (f) Calculated $Q_{f}$ with different $x_{1}$ by using $V_{o c-f}\left(C_{f}+C_{x l}\right)$.

(a)

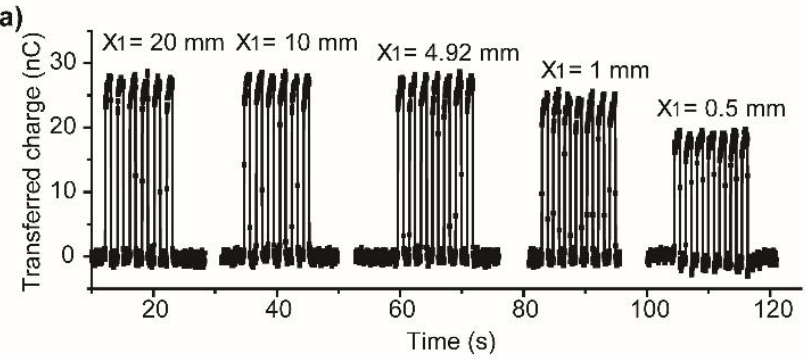

(b)

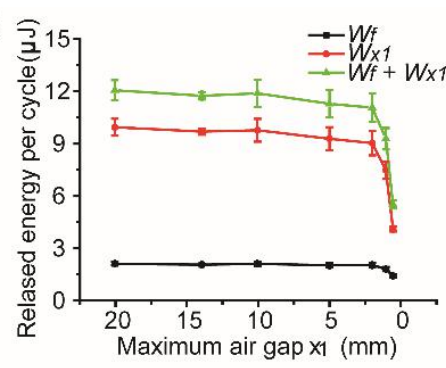

Fig. 4. (a) Measured transferred charge amounts and (b) calculated released energy per cycle from the TENG with different $x_{1}$. 
(a)

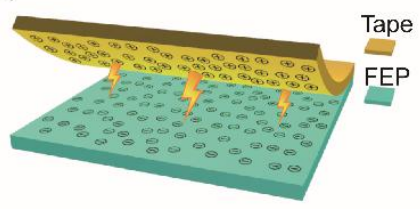

(b) $\sum_{-3000} \rightarrow$-Before test

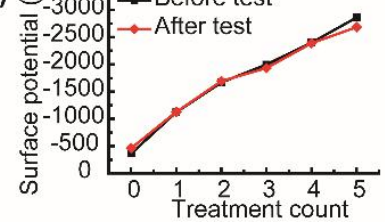

Fig. 5. Charge the FEP film with the tape-peeling method. (a) The schematic of charging the FEP film with tape. (b) Increased surface potential of the $4 \times 4 \mathrm{~cm}^{2}$ sized FEP film with increasing tape-peeling treatment counts, before and after the linear motor test.
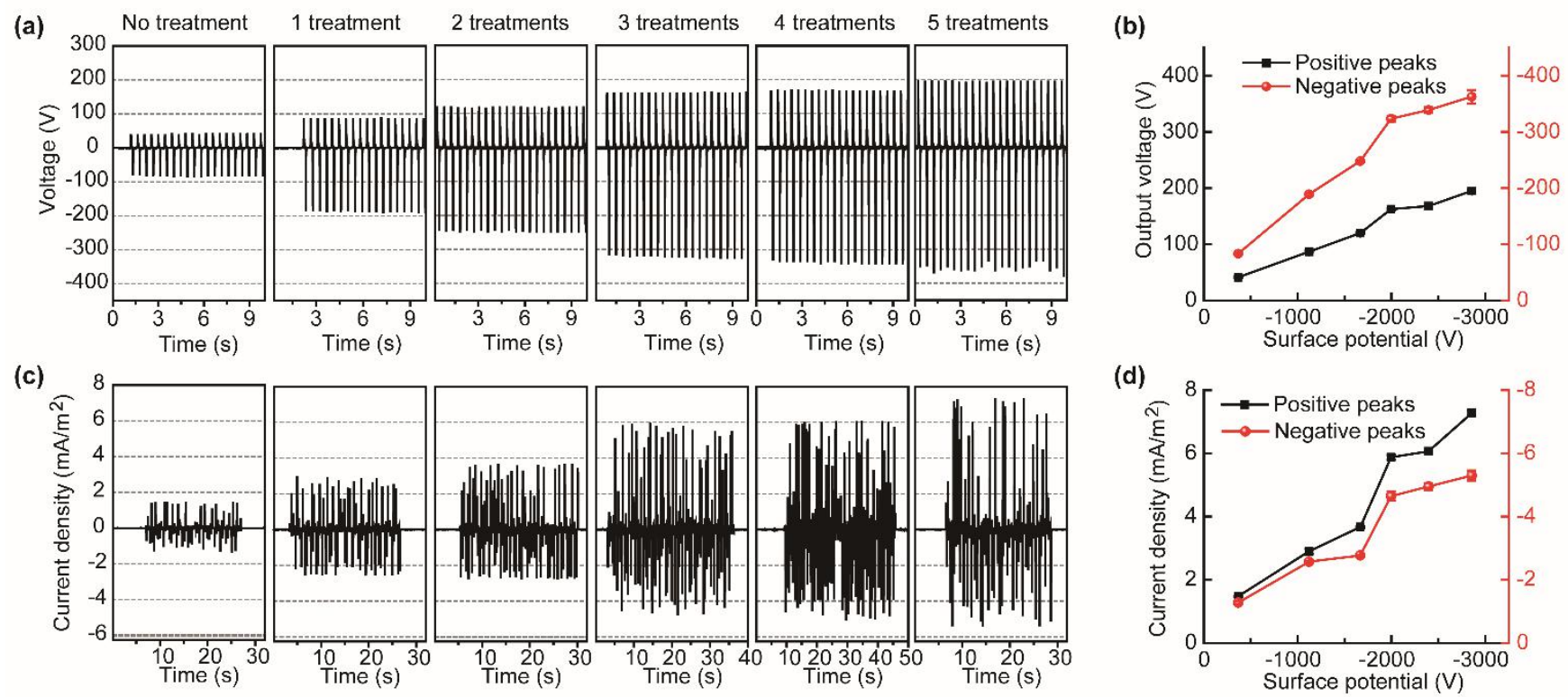

Fig. 6. Improvement of the TENG in output voltages and currents. (a) Increased output voltages of the $4 \times 4 \mathrm{~cm}^{2}$ sized TENG with the FEP film treated by the tape with increasing counts (from left to right: $0 \sim 5$ counts). (b) Statistic results of the positive and negative voltage peak values. (c) Increased current density of the TENG with increasing treatment counts. (d) Statistic results of the positive and negative current density peak values. 

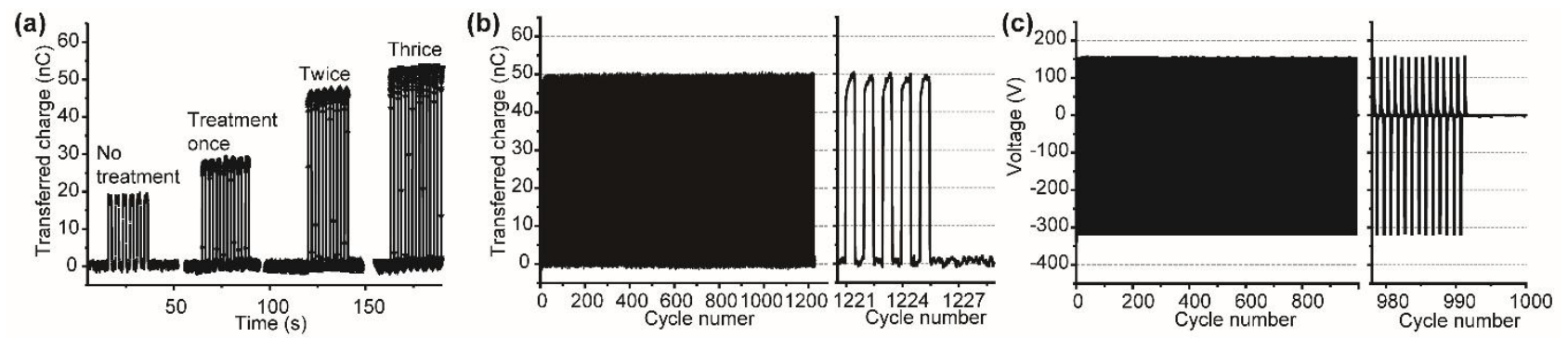

Fig. 7. (a) Increased transferred charge amount of the TENG by increasing tape treatment counts.

(b) Durability of the TENG in transferred charge amount. (c) Durability of the TENG in output voltage.
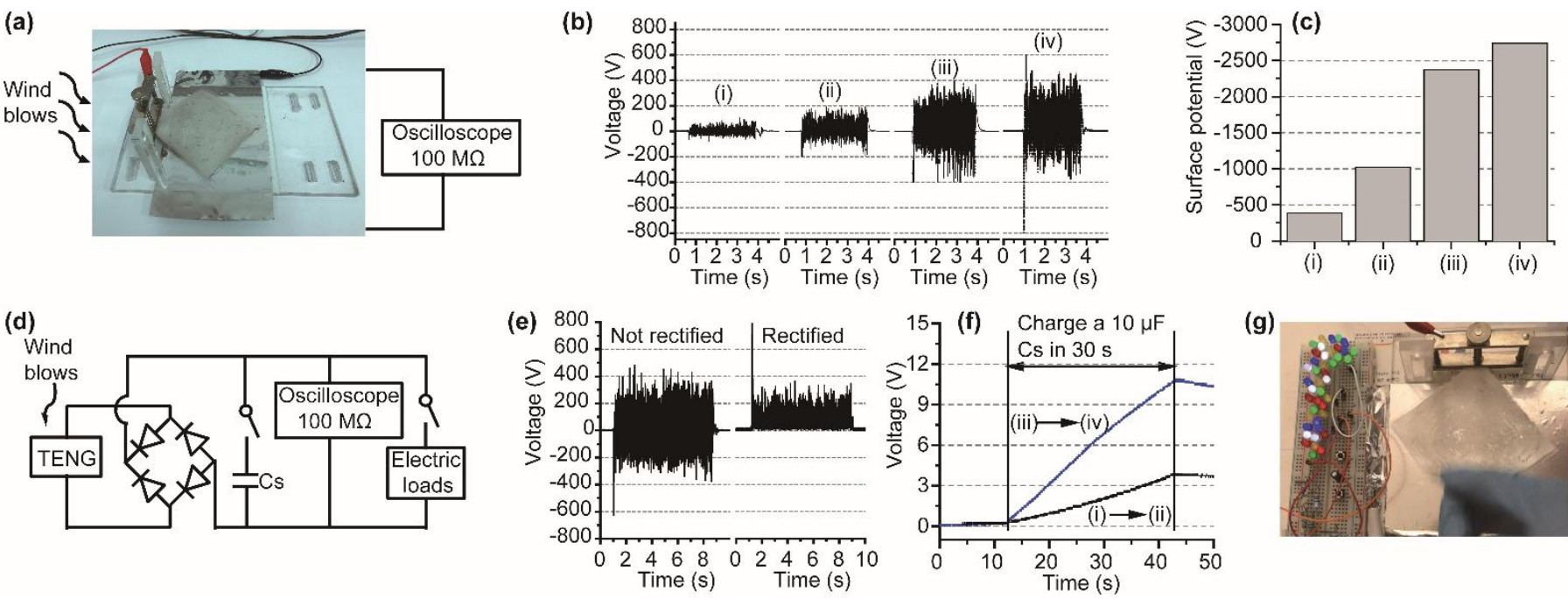

Fig. 8. Demonstrations of energy harvesting by the TENG from wind and human motions. (a)

The photograph of the wind-driven TENG with an $8 \mathrm{~cm} \times 8 \mathrm{~cm}$ sized FEP film, and the illustration of its working process. (b) Output voltages of the TENG with FEP film holding different surface potentials. (c) Measured surface potentials of the FEP film corresponding to the output voltages in (b). (d) The circuit diagram of the TENG driven by wind to charge a capacitor (Cs) or to power electric loads. (e) Output voltage of the TENG before and after rectification, driven by wind. (f) Charging curves of the Cs charged by the TENG before and after tape treatment. (g) The photograph showing that 42 LEDs were directly lighted up by the TENG when the FEP film was slightly and slowly flapped by a human hand. 


\section{Vitae}

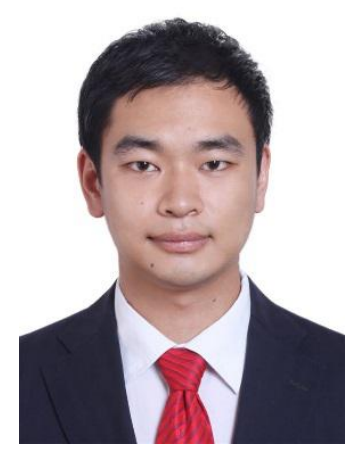

Hanlu Zhang received his M.S. degree (2016) of Materials Science from Zhengzhou University, under the supervision of Prof. Lin Dong, and he studied at the Beijing Institute of Nanoenergy and Nanosystems as a joint training master candidate during 2013 2015, co-supervised by Prof. Caofeng Pan. Now he is a CSC sponsored Ph.D. candidate directed by Prof. Jinbo Bai at Ecole CentraleSupélec of the University of Paris-Saclay. His current research interests are focused on preparations and characterizations of electrets and triboelectric nanogenerators.

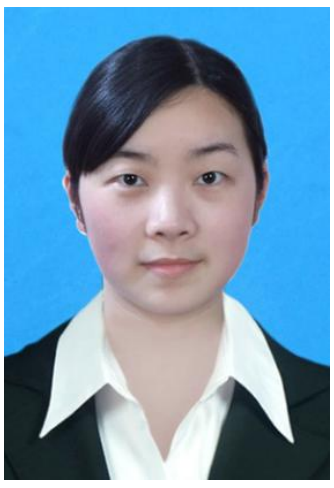

Shan Feng received her B.S. and M.S. degree in Materials Science and Engineering from China University of Geosciences, under the supervision of Prof. Jin TAN. Now she is a CSC sponsored Ph.D. candidate directed by Prof. Jinbo Bai at Ecole CentraleSupélec of the University of Paris-Saclay. Her research mainly focuses on dielectric material synthesis and characterization, fabrication of triboelectric nanogenerators.

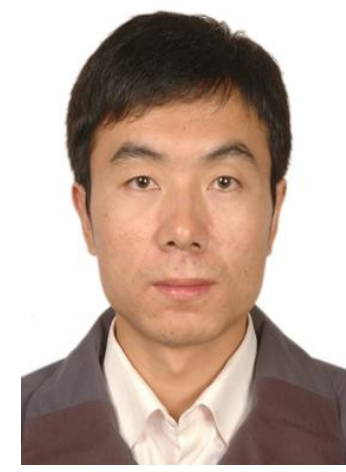

Delong He received his $\mathrm{PhD}$ degree in materials science from Ecole Centrale Paris (France) in 2010. He conducted Post-doc research at CEA-Saclay in 2011 and then at CNRS MSSMat laboratory from 2012 to 2013. Since 2016, he is permanent research engineer of Ecole CentraleSupélec (France). His research interests are multifunctional composite materials and energy.

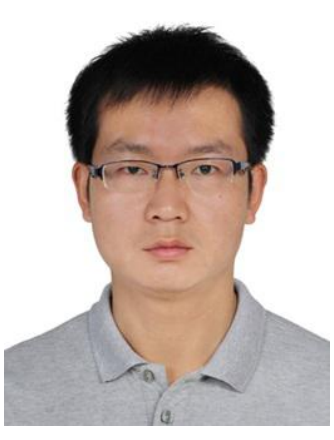

Yiguo Xu received his M.S. degree from Fudan University at 2013. As a master degree candidate, he did researches on DFT calculations and Molecular dynamics simulations. Now he is a Ph.D. candidate supervised by Prof. Jinbo Bai at Ecole CentraleSupélec of the University of Paris-Saclay. His current interest area is CNTs growth by Chemical Vapor Deposition method. 


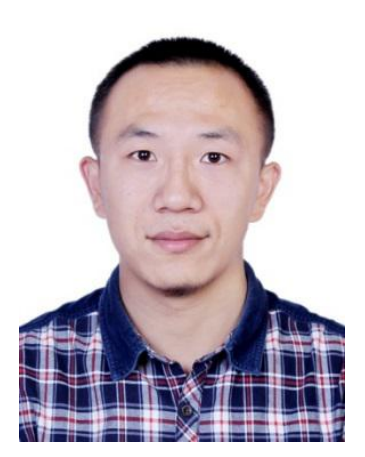

Minhao Yang was born in Shanxi, China in 1990. He received his B.S. degree in Materials Science and Engineering from Zhejiang Sci-Tech University (China) in 2013, and his M.S. degree in Polymer Science and Engineering from University of Science and Technology Beijing (China) under the supervision of Prof. Zhi-Min Dang in 2015. Then he joined the CNRS laboratory of Prof. Jinbo Bai at CentraleSupélec, Université Paris-Saclay (France) to pursue his PhD degree with the financial support from CSC in 2015. His research interests mainly focus on the rational design of one-dimensional carbon nano-hybrids with the core@shell structure for dielectric nanocomposites application.

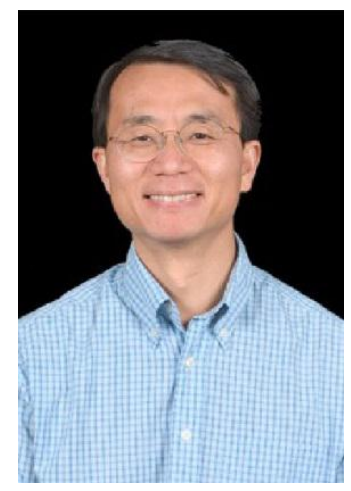

Jinbo Bai received his Master degree of Solid Mechanics from Xi'an Jiaotong University in 1985 and Ph.D. degree of Science of Materials from Ecole Centrale Paris in 1991. He was lecturer of Mechanics of Materials in Northwestern Polytechnic University 1985-1987 and CNRS Researcher then Director of research in Lab. MSSMAT, UMR8579 of Ecole Centrale Paris since 1991. His research interest cover from nuclear materials Zr\&Ti, hydrogen storage and embrittlement, composites materials, micromechanical modelling, finite element simulations, carbon nanomaterials' syntheses, characterizations and applications, structural and multi-functional nano/microcomposites, hybrid fillers, heat management, energy storage and green energy in general. 